Supplement of

\title{
Measurement report: The chemical composition and temporal variability of aerosol particles at Tuktoyaktuk, Canada during the Year of Polar Prediction Special Observing Period
}

John MacInnis et al.

Correspondence to: Rachel Ying-Wen Chang (rachel.chang@dal.ca) 
Table S1. Masses and detection frequencies of ions and metals in fine (PM 2.5$)$ and coarse (PM102.5) aerosol filter samples from Tuktoyaktuk. Means were calculated using masses equal to or greater than the detection limit (DL).

\begin{tabular}{|c|c|c|c|c|c|}
\hline & $\begin{array}{c}\text { Particle } \\
\text { Size } \\
\end{array}$ & $\begin{array}{l}\text { DL } \\
(\mu g)\end{array}$ & $\begin{array}{c}\text { Mean } \\
(\mu \mathrm{g})\end{array}$ & $\begin{array}{c}\text { Range } \\
(\mu g)\end{array}$ & $\begin{array}{c}\text { Frequency } \\
(\%)\end{array}$ \\
\hline \multicolumn{6}{|l|}{ Ions } \\
\hline \multirow{2}{*}{$\mathrm{F}^{-}$} & $\mathrm{PM}_{2.5}$ & \multirow{2}{*}{2.4} & $<2.4$ & $<2.4$ & 0 \\
\hline & $\mathrm{PM}_{10-2.5}$ & & 2.5 & $<2.4-2.7$ & 25 \\
\hline \multirow{2}{*}{$\mathrm{Cl}^{-}$} & $\mathrm{PM}_{2.5}$ & \multirow{2}{*}{0.020} & 0.45 & $<0.020-1.2$ & 62 \\
\hline & $\mathrm{PM}_{10-2.5}$ & & $<0.020$ & $<0.020$ & 0 \\
\hline \multirow{2}{*}{$\mathrm{NO}_{2}^{-}$} & $\mathrm{PM}_{2.5}$ & \multirow{2}{*}{0.050} & $<0.050$ & $<0.050$ & 0 \\
\hline & $\mathrm{PM}_{10-2.5}$ & & 0.10 & $<0.050-0.10$ & 12 \\
\hline \multirow{2}{*}{$\mathrm{Br}^{-}$} & $\mathrm{PM}_{2.5}$ & \multirow{2}{*}{0.090} & $<0.090$ & $<0.090$ & 0 \\
\hline & $\mathrm{PM}_{10-2.5}$ & & 0.19 & $<0.090-0.19$ & 25 \\
\hline \multirow{2}{*}{$\mathrm{NO}_{3}^{-}$} & $\mathrm{PM}_{2.5}$ & \multirow{2}{*}{0.39} & 0.40 & $<0.39-0.40$ & 25 \\
\hline & $\mathrm{PM}_{10-2.5}$ & & 0.42 & $<0.39-0.42$ & 12 \\
\hline \multirow{2}{*}{$\mathrm{PO}_{4}^{3-}$} & $\mathrm{PM}_{2.5}$ & \multirow{2}{*}{0.12} & 0.61 & $<0.12-0.87$ & 25 \\
\hline & $\mathrm{PM}_{10-2.5}$ & & 1.1 & $<0.12-1.4$ & 37 \\
\hline \multirow{2}{*}{$\mathrm{SO}_{4}{ }^{2-}$} & $\mathrm{PM}_{2.5}$ & \multirow{2}{*}{0.17} & 0.48 & $<0.17-1.2$ & 87 \\
\hline & $\mathrm{PM}_{10-2.5}$ & & $<0.17$ & $<0.17$ & 0 \\
\hline \multirow{2}{*}{$\mathrm{NH}_{4}{ }^{+}$} & $\mathrm{PM}_{2.5}$ & \multirow{2}{*}{0.19} & 0.21 & $<0.19-0.21$ & 12 \\
\hline & $\mathrm{PM}_{10-2.5}$ & & $<0.19$ & $<0.19$ & 0 \\
\hline \multirow{2}{*}{$\mathrm{Ca}^{2+}$} & $\mathrm{PM}_{2.5}$ & \multirow{2}{*}{0.28} & 0.72 & $<0.28-0.95$ & 25 \\
\hline & $\mathrm{PM}_{10-2.5}$ & & $<0.28$ & $<0.28$ & 0 \\
\hline \multicolumn{6}{|l|}{ Metals } \\
\hline \multirow{2}{*}{$\mathrm{Al}$} & $\mathrm{PM}_{2.5}$ & \multirow{2}{*}{0.030} & 0.089 & $0.030-0.3$ & 100 \\
\hline & $\mathrm{PM}_{10-2.5}$ & & 0.054 & $<0.030-0.078$ & 75 \\
\hline \multirow{2}{*}{$\mathrm{Fe}$} & $\mathrm{PM}_{2.5}$ & \multirow{2}{*}{0.13} & 0.26 & $<0.13-0.26$ & 25 \\
\hline & $\mathrm{PM}_{10-2.5}$ & & 0.16 & $<0.13-0.16$ & 12 \\
\hline \multirow{2}{*}{$\mathrm{Ag}$} & $\mathrm{PM}_{2.5}$ & \multirow{2}{*}{0.0024} & $<0.0024$ & $<0.0024$ & 0 \\
\hline & $\mathrm{PM}_{10-2.5}$ & & 0.0026 & $<0.0024-0.0029$ & 25 \\
\hline \multirow{2}{*}{$\mathrm{Ti}$} & $\mathrm{PM}_{2.5}$ & \multirow{2}{*}{0.0030} & 0.0052 & $<0.0030-0.0074$ & 25 \\
\hline & $\mathrm{PM}_{10-2.5}$ & & $<0.0030$ & $<0.0030$ & 0 \\
\hline$C_{0}$ & $\mathrm{PM}_{2.5}$ & & $<0.012$ & $<0.012$ & 0 \\
\hline $\mathrm{Cu}$ & $\mathrm{PM}_{10-2.5}$ & 0.012 & 0.013 & $<0.012-0.013$ & 12 \\
\hline & $\mathrm{PM}_{2.5}$ & & 0.029 & $<0.012-0.029$ & 12 \\
\hline $\mathrm{Sb}$ & $\mathrm{PM}_{10-2.5}$ & 0.012 & 0.021 & $<0.012-0.021$ & 12 \\
\hline $\mathrm{Ba}$ & $\mathrm{PM}_{2.5}$ & 0010 & $<0.019$ & $<0.019$ & 0 \\
\hline Ba & $\mathrm{PM}_{10-2.5}$ & 0.019 & 0.047 & $<0.019-0.072$ & 37 \\
\hline & $\mathrm{PM}_{2.5}$ & & 0.011 & $<0.0080-0.011$ & 25 \\
\hline $\mathrm{Zn}$ & $\mathrm{PM}_{10-2.5}$ & 0.0080 & 0.011 & $<0.0080-0.012$ & 37 \\
\hline
\end{tabular}


Table S2. An overview of formulas used for mass reconstruction analysis, adapted from Bari and Kindzierski (2017). The chemical masses of ions and metals were used in formulas.

\begin{tabular}{cl}
\hline Component & \multicolumn{1}{c}{ Formula } \\
\hline$\left(\mathrm{NH}_{4}\right)_{2} \mathrm{SO}_{4}$ & {$\left[\left(\mathrm{NH}_{4}\right)_{2} \mathrm{SO}_{4}\right]=\left[\mathrm{SO}_{4}{ }^{2-}\right]+\left[\mathrm{NH}_{4}{ }^{+}\right]-0.29\left[\mathrm{NO}_{3}-\right]$} \\
$\mathrm{NH}_{4} \mathrm{NO}_{3}$ & {$\left[\mathrm{NH}_{4} \mathrm{NO}_{3}\right]=1.29\left[\mathrm{NO}_{3}\right]$} \\
$\mathrm{NaCl}$ & {$[\mathrm{NaCl}]=1.65\left[\mathrm{Cl}^{-}\right]$} \\
Mineral Dust $(\mathrm{MD})$ & {$[\mathrm{MD}]=2.20[\mathrm{Al}]+1.63\left[\mathrm{Ca}^{2+}\right]+2.42[\mathrm{Fe}]+1.94[\mathrm{Ti}]$} \\
Metal Oxides (MO) & {$[\mathrm{MO}]=1.25[\mathrm{Cu}]+1.24[\mathrm{Zn}]+1.07[\mathrm{Ag}]+1.20[\mathrm{Sb}]+1.12[\mathrm{Ba}]$} \\
Particle-bound water (PBW) & {$[\mathrm{PBW}]=0.32\left[\mathrm{SO}_{4}{ }^{2-}+\mathrm{NH}_{4}{ }^{+}\right]$} \\
Unclassified Inorganic (UI) & {$[\mathrm{UI}]=\left[\mathrm{F}^{-}\right]+\left[\mathrm{Br}^{-}\right]+\left[\mathrm{PO}_{4}{ }^{-}\right]+\left[\mathrm{NO}_{2}{ }^{-}\right]$} \\
\hline
\end{tabular}



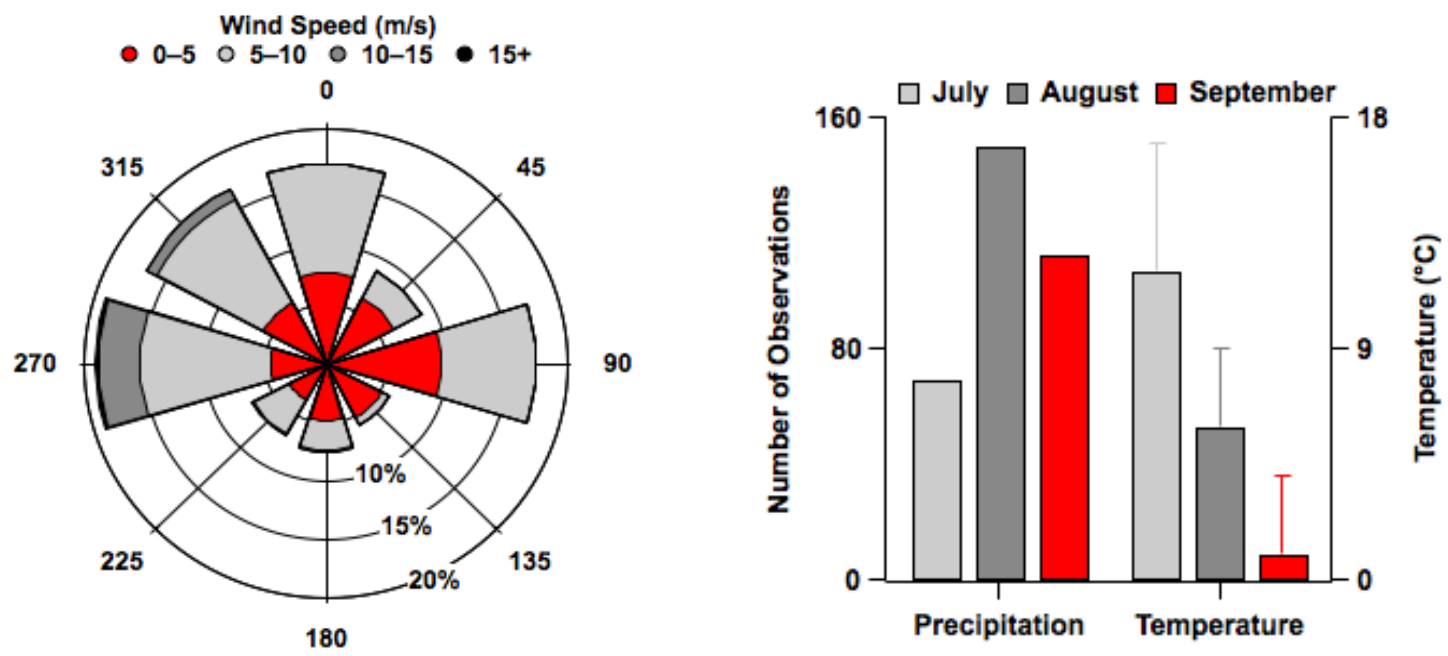

Figure S1. Wind speed and direction, frequency of precipitation, and air temperature at the Tuktoyaktuk airport. Wind speed and direction and air temperature are based on hourly data beginning at 0:00 on 18 July to 23:00 on 13 September (local time). Error bars associated with air temperature measurements are standard deviations. The frequency of precipitation is based on hourly data from 1 July to 30 September 2018 during the hours of 7:00 to 23:00. Precipitation refers to both liquid and solid hydrometeors (i.e., drizzle, rain, snow, hail). The data presented in this figure can be found in Historical Climate Archives (Environment and Climate Change Canada, 2020). 
18 July 2018

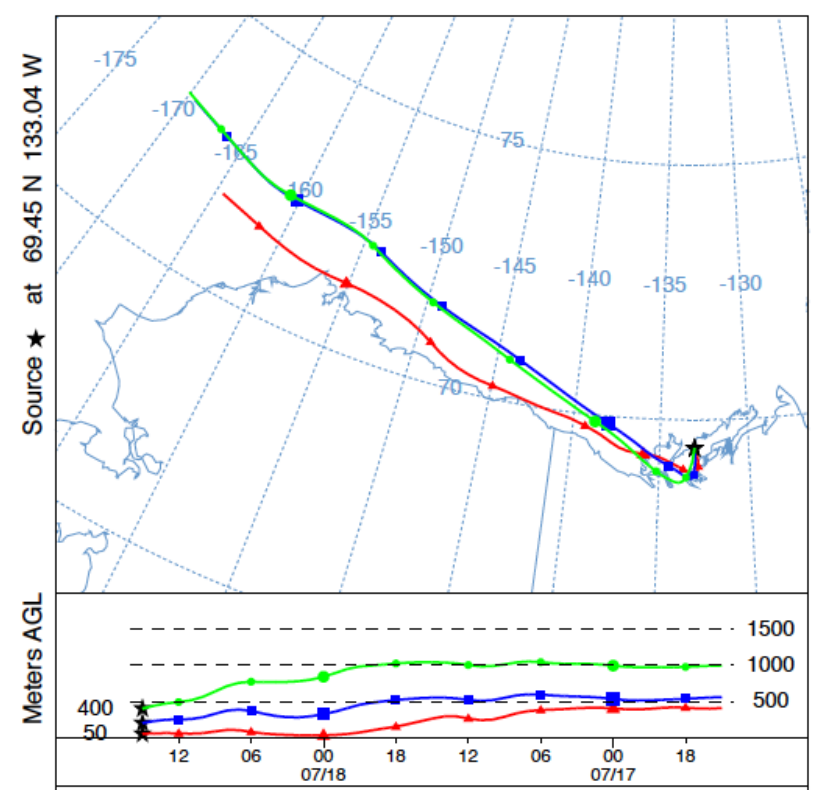

3 August 2018

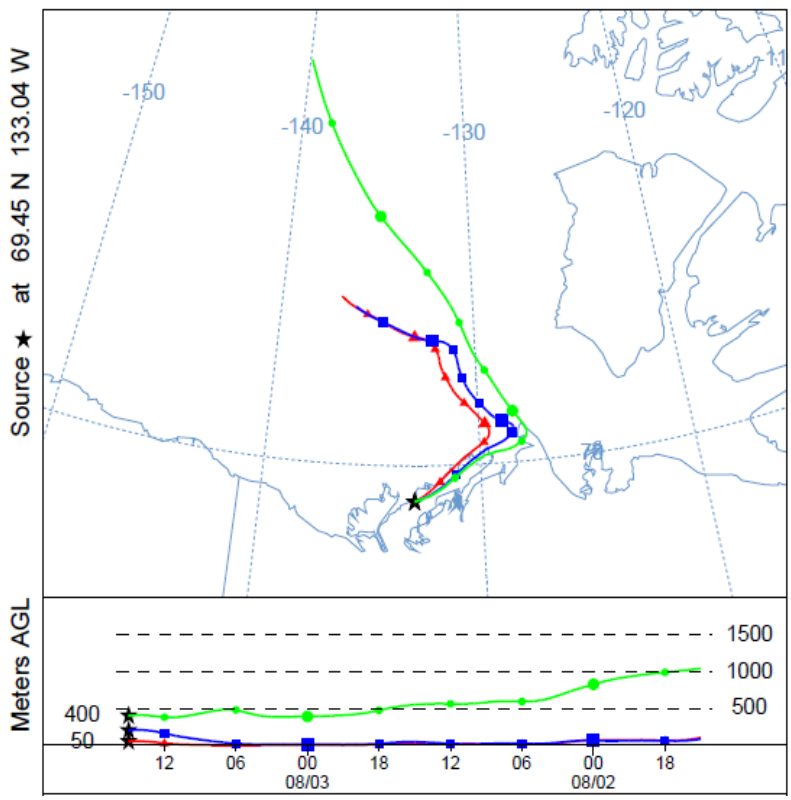

26 July 2018

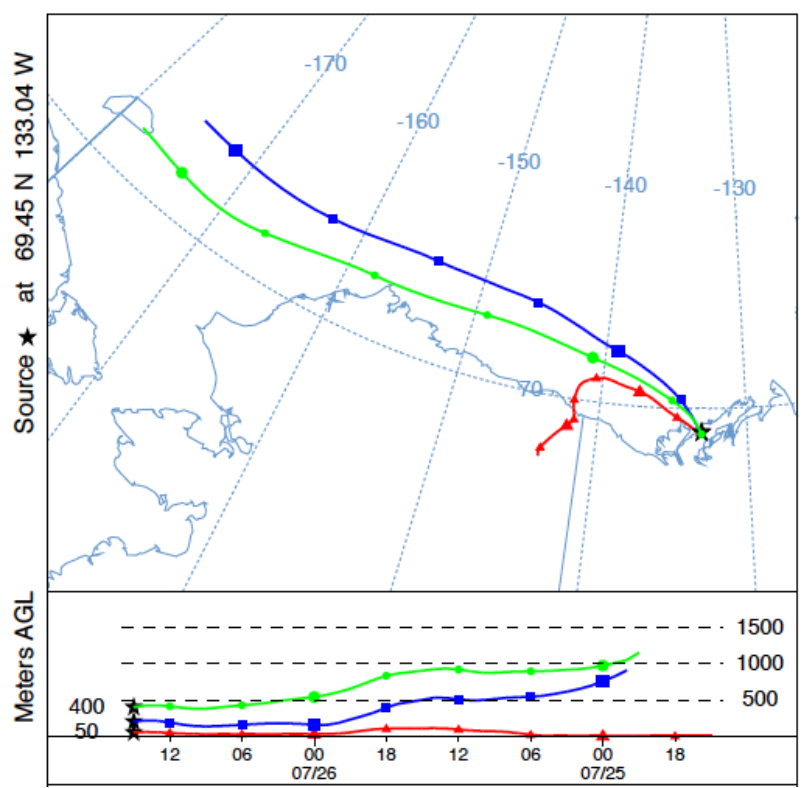

11 August 2018

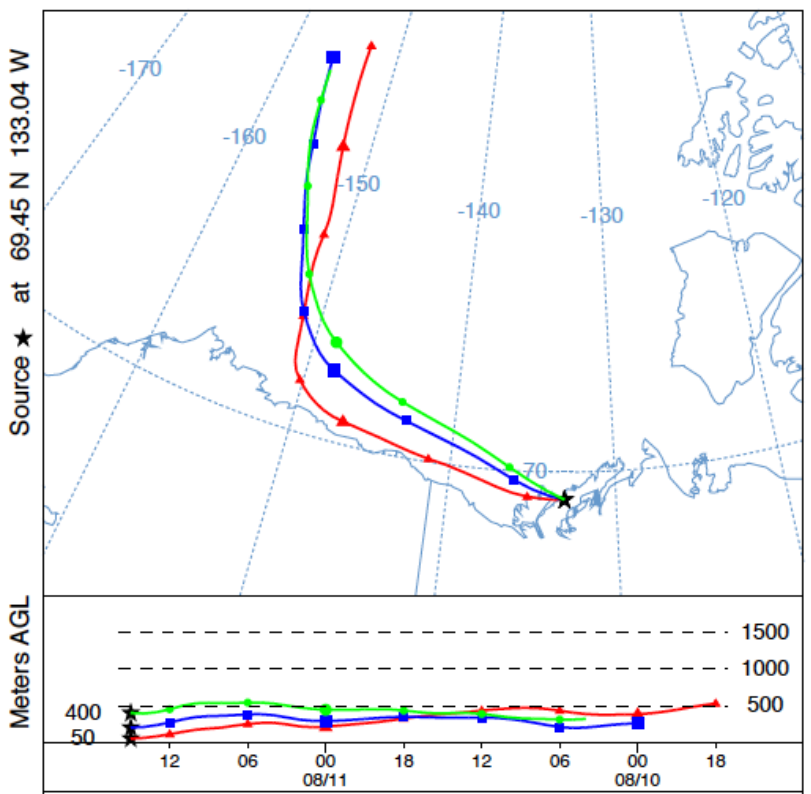

Figure S2. Air mass back trajectories ( $48 \mathrm{~h}$ ) during 18 July to 11 August using the HYSPLIT Model (National Oceanic and Atmospheric Administration, https://www.ready.noaa.gov/HYSPLIT_traj.php) with North American Regional Reanalysis meteorology at heights of 50,200, and $400 \mathrm{~m}$ above ground level. 
19 August 2018

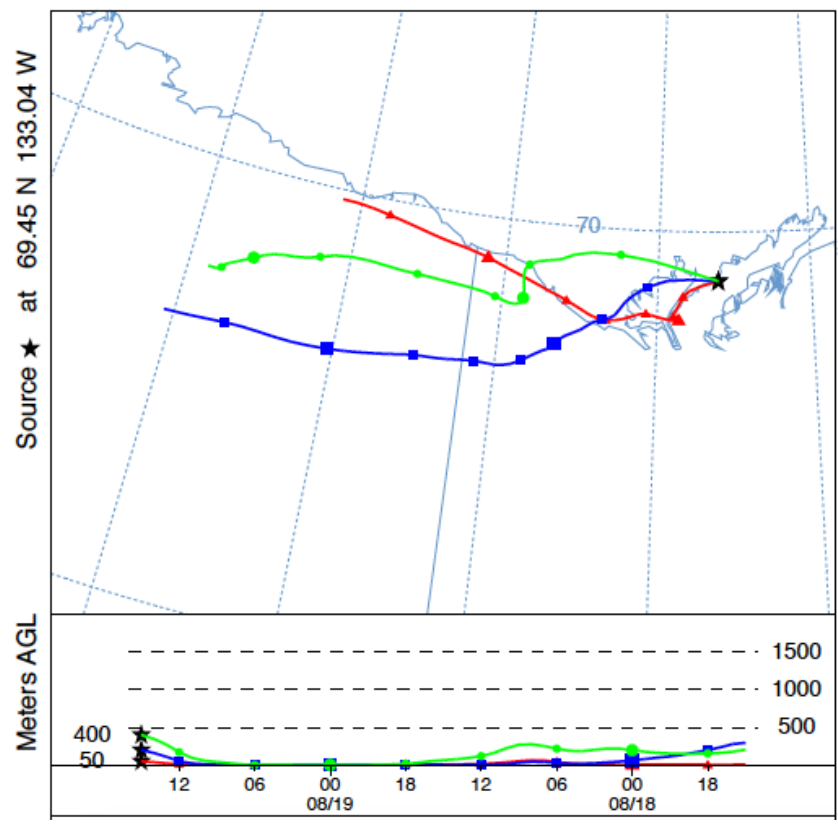

4 September 2018

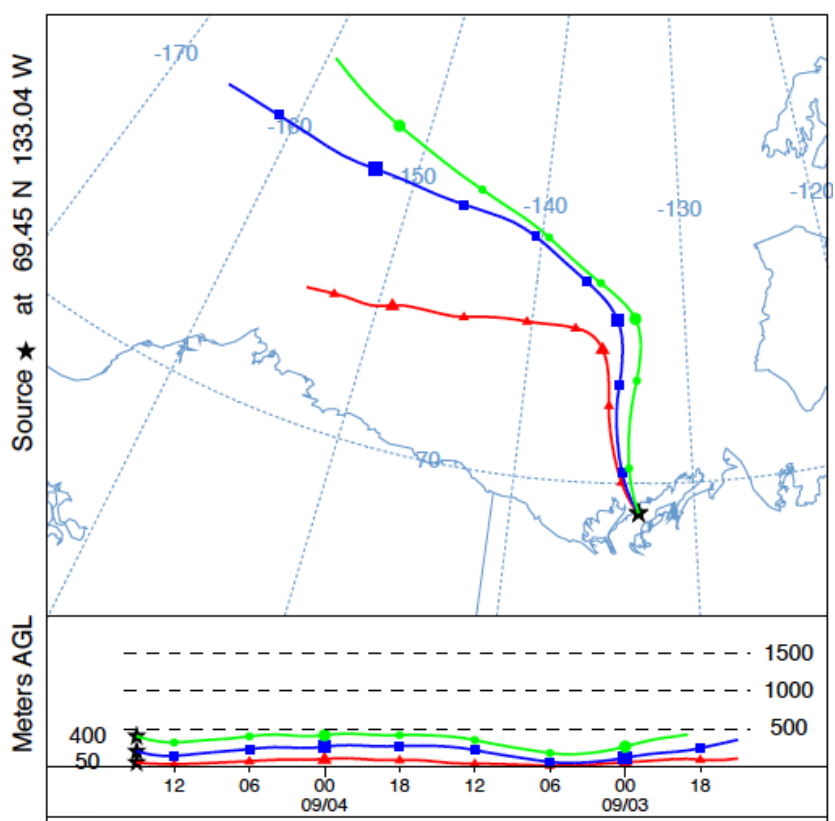

27 August 2018

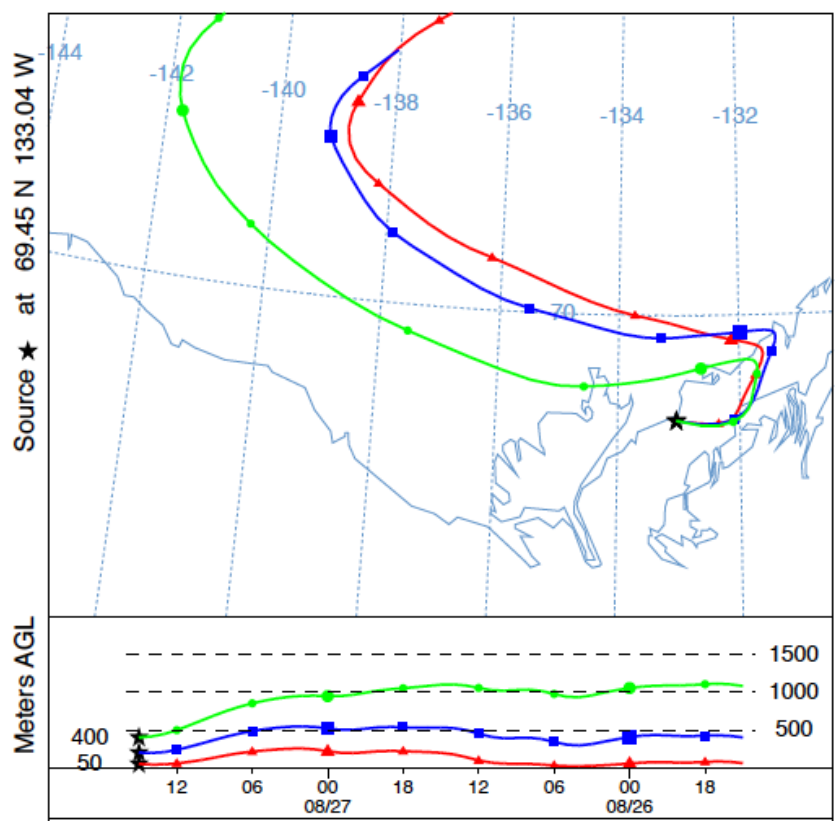

12 September 2018

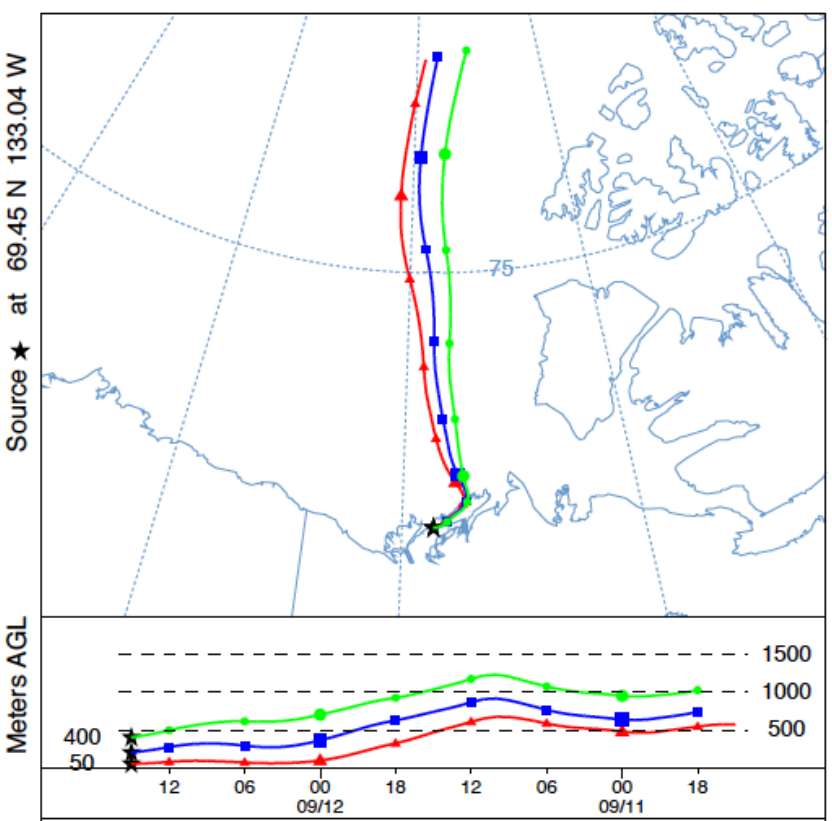

Figure S3. Air mass back trajectories ( $48 \mathrm{~h}$ ) during 19 August to 12 September using the HYSPLIT Model (National Oceanic and Atmospheric Administration, https://www.ready.noaa.gov/HYSPLIT_traj.php) with North American Regional Reanalysis meteorology at heights of 50, 200, and $400 \mathrm{~m}$ above ground level. 

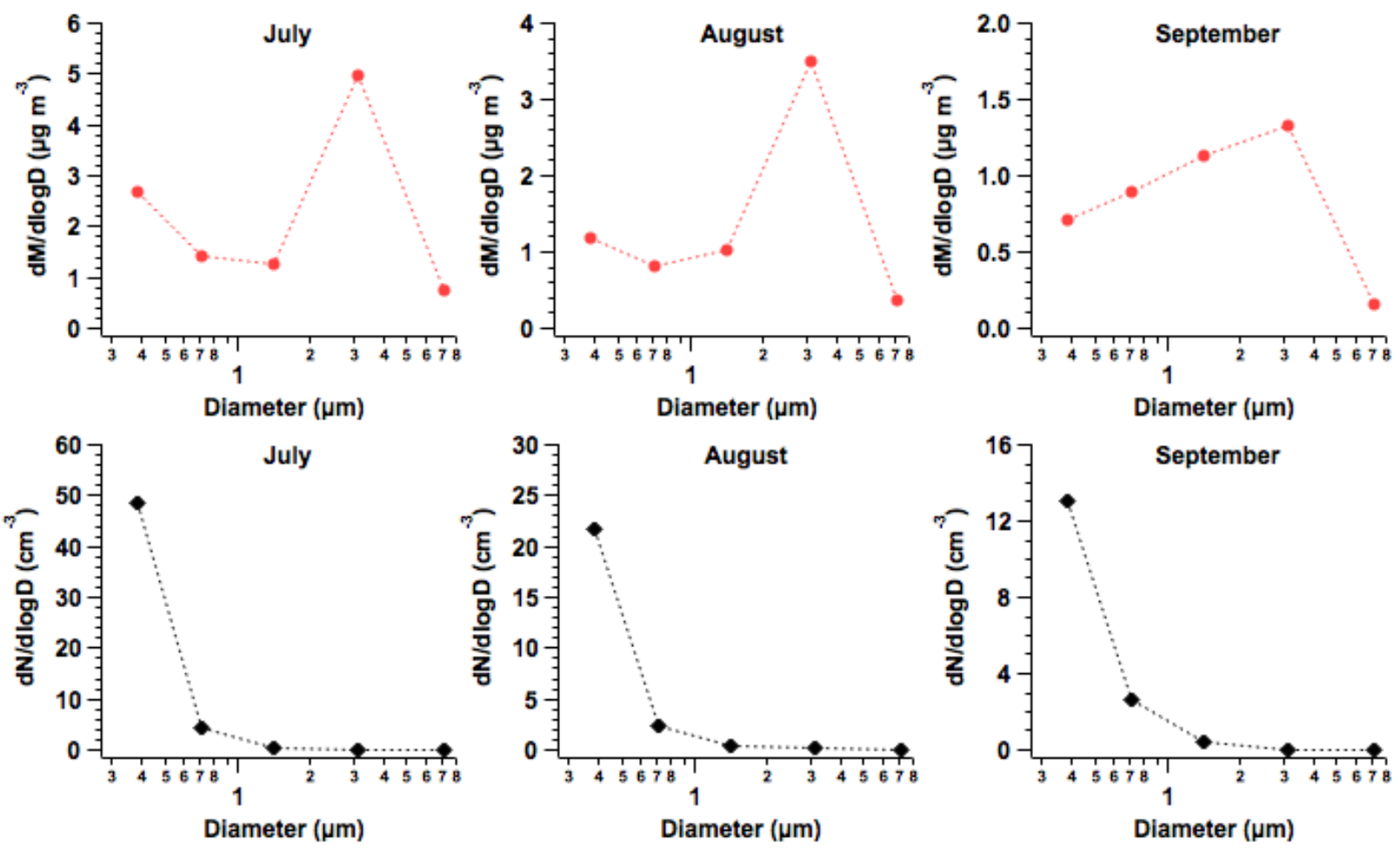

Figure S4. Average number (bottom row) and mass (top row) size distributions of aerosol particles collected from Tuktoyaktuk during July-September 2018. The x-axis refers to the geometric mean bin diameters for the optical particle counter. 


\section{References}

Bari, M.A., and Kindzierski, W.B.: Ambient fine particulate matter $\left(\mathrm{PM}_{2.5}\right)$ in Canadian oil sands communities: Levels, sources and potential human health risk, Sci. Total Environ., 595, 828-838, doi:10.1016/j.scitotenv.2017.04.023, 2017.

Environment and Climate Change Canada. 2020. Historical Climate Data: https://climate.weather.gc.ca/historical_data/search_historic_data_stations_e.html?searchTy pe $=$ stnName \& timeframe $=1 \&$ txtStationName $=$ tuktoyaktuk \&searchMethod=contains $\& o p t L i$ mit=yearRange $\&$ StartYear=1840\&EndYear=2020\&Year=2020\&Month=9\&Day=7\&selRo wPerPage $=25$, last access: 15 September 2020. 